\title{
Analysis on Aircraft Brake Squeal Problem Based on Finite Element Method
}

\author{
Ming Zhang, ${ }^{1}$ Ran Xu, ${ }^{1}$ and Hong Nie ${ }^{1,2}$ \\ ${ }^{1}$ Key Laboratory of Fundamental Science for National Defense-Advanced Design Technology of Flight Vehicle, \\ Nanjing University of Aeronautics and Astronautics, Nanjing 210016, China \\ ${ }^{2}$ State Key Laboratory of Mechanics and Control of Mechanical Structures, Nanjing University of Aeronautics and Astronautics, \\ Nanjing 210016, China
}

Correspondence should be addressed to Ming Zhang; zhm6196@nuaa.edu.cn

Received 15 February 2017; Revised 5 April 2017; Accepted 19 April 2017; Published 28 May 2017

Academic Editor: Hikmat Asadov

Copyright (c) 2017 Ming Zhang et al. This is an open access article distributed under the Creative Commons Attribution License, which permits unrestricted use, distribution, and reproduction in any medium, provided the original work is properly cited.

\begin{abstract}
Brake squeal phenomenon is a problem that has been long studied using multiple methods and theories. Finite Element Method (FEM) has been applied to the study of brake squeal problem. First, a disc brake model has been established. Complex mode theory has been applied to the mode analysis and unstable vibration modes can be extracted subsequently. The form of unstable vibration mode has been studied. Then, transient dynamic simulation using explicit dynamic method has been performed. Response in both time and frequency domain has been analyzed. Two methods have been compared, considering accuracy and calculation consumption. Then, the effect of different parameters such as coefficient of friction, stiffness, and brake force fluctuation frequency on squeal phenomenon has been analyzed. It can be found that coefficient of friction and the brake stiffness have a positive correlation with the extent of brake squeal phenomenon, while the frequency of brake force fluctuation should remain as low as possible. Afterwards, a ring-shaped layer of viscoelastic damping material is constrained to outer margin of the stator to restrain the unstable modal. This method can change the vibration nature and improve the brake squeal problem.
\end{abstract}

\section{Introduction}

Along with the rising requirement of comfort in the field of civil aviation, brake noise problem has gained great attention because it considerably affects passengers' convenience [1-3]. Aircraft brake system generates vibration during its normal working process, followed by noise inevitably. It has been over 60 years since foreign researchers began working on brake squeal problem, while domestic researchers merely initiated their study in 1980s. In terms of the classification of squeal problem, a universal standard has not yet been put forth. However, researchers agree on naming different types of brake noise according to their respective vibration frequency. Most of the researchers tend to divide squeal phenomenon into three kinds. First type of brake noise possesses a frequency range from $100 \mathrm{~Hz}$ to $1 \mathrm{kHz}$, which is relatively low. This type of brake noise comes from the stickslip motion between friction surfaces [4]. Applying other materials whose coefficient of friction is stable about slip speed could ease this type of brake noise effectively. Second type of brake noise is generated due to the occurrence of modal coupling among brake parts, with a frequency range between $1 \mathrm{kHz}$ and $3 \mathrm{kHz}$. The third type of brake noise is generally caused by modal response of brake discs, whose frequency is between $3 \mathrm{kHz}$ and $15 \mathrm{kHz}$. The last two types of brake noise are often named as "squeal," which has always been an important issue in the field of brake noise research [5-8].

Brake squeal phenomenon brings tremendous oscillation load to both brake mechanism and landing gear structure, which greatly interferes with pilot's regular flight control and passengers' comfort. Serious brake squeal may even damage the entire brake system [9]. As a result, it is quite necessary to study aircraft brake squeal problem thoroughly.

In the field of experiment, many researchers have found that it is very hard to replicate brake squeal in the lab [10]. Moreover, noise generated in the lab does not have a stable frequency and sound pressure level [11-14]. During 


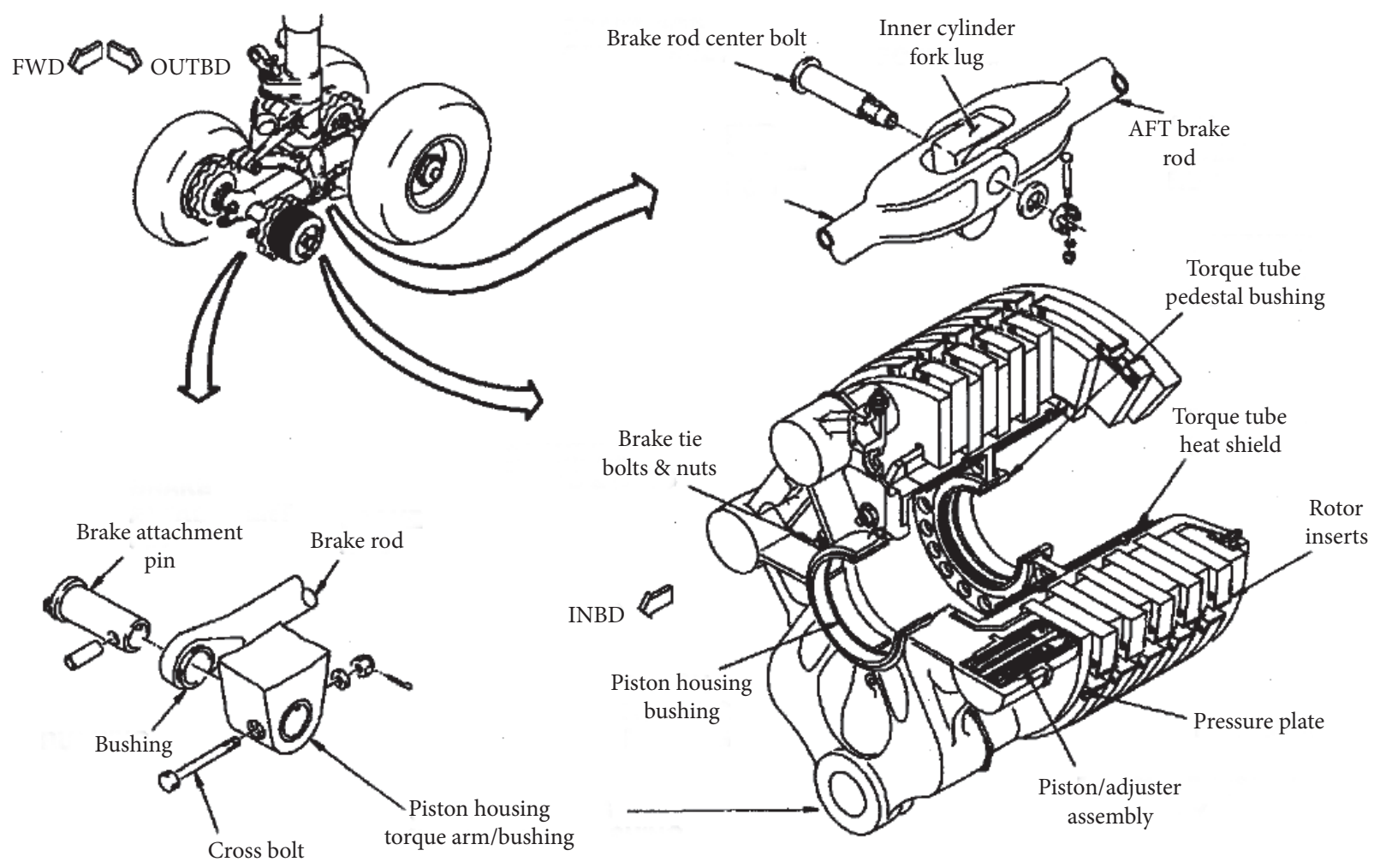

FIGURE 1: Schematic figure of aircraft brake system.

experiment, Oberst and Lai discovered that even brake force, speed, and temperature are stable; the sound pressure level of brake force still has a considerable straggling tendency [15]. Beloiu and Ibrahim discovered that even during one single experiment progress, there can still be an undeniable straggling tendency in sound pressure level, contact force of friction surface, and coefficient of friction [16]. So far, no clear result can be concluded from the experiments.

As the theoretical research proceeds over time, four main theories dealing with brake squeal problem have been put forward by researchers. They are stick-slip theory, lock-slip theory, negative slope of friction force theory, and modal coupling theory. The previous two theories emphasize stickslip property; the latter two theories concentrate on structural instability [17]. In all, researchers have been studying the cause and influence factor of brake squeal problem for a long time. But unfortunately, a complete and universal theory to handle brake squeal problem has not been invented yet [18]. However, there is no doubt that modal coupling and modal response method show promising prospect in coping with brake squeal problem.

Combining with previous researchers' experience, an FEM model of an actual aircraft brake system has been constructed. Complex mode analysis is thoroughly introduced and applied to the aircraft brake model. Complex eigenvalues are extracted and analyzed. The vibration property of certain typical unstable frequencies has been analyzed. With the help of explicit dynamic analysis, transient dynamic analysis has been performed on the aircraft brake model. The displacement response in both time domain and frequency domain has been acquired. Several influence factors are studied by applying complex mode method.

\section{Aircraft Disc Brake Modeling}

Schematic figure of a typical aircraft disc brake is shown in Figure 1. It mainly consists of pistons, torque tubes, brake rods, and a set of brake discs that stack alternatively. Hydraulic system provides pressure for the brake procedure. Pistons empowered by hydraulic system push a set of ganged stators against a set of ganged rotors, so brake force can be generated by friction between brake discs. Brake force is transferred to the entire landing gears structure through wheel axis and piston houses. Among the primary brake mechanisms, carbon brake discs carry out the most crucial functions of the brake system, which is friction. Carbon brake discs enable the aircraft to completely stop moving while the plane is taking off and landing; this makes the discs key components in the brake system.

A simplified brake model is built according to the FEM modeling principles. Subsequent improvements are conducted by setting up contact, friction, mesh, and element properties. This reliable model is the foundation stone for further analysis.

2.1. Physical Modeling. Brake discs model are currently applied in actual type of civil aircraft. The detailed CAD models are given in CATIA software. All the parts are made of carbon composite material, whose Young's modulus is $80 \mathrm{GPa}$, density is $1800 \mathrm{~kg} / \mathrm{m}^{3}$, and coefficient of friction on 


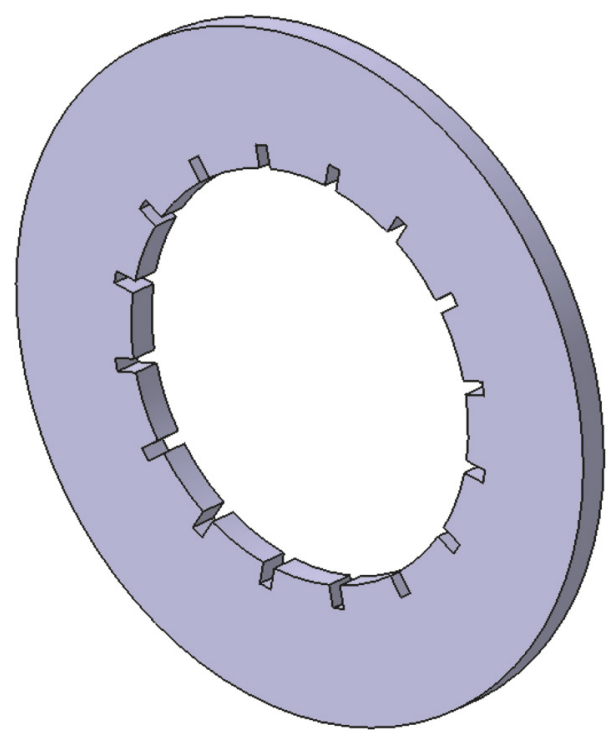

Figure 2: Simplified model of stator.

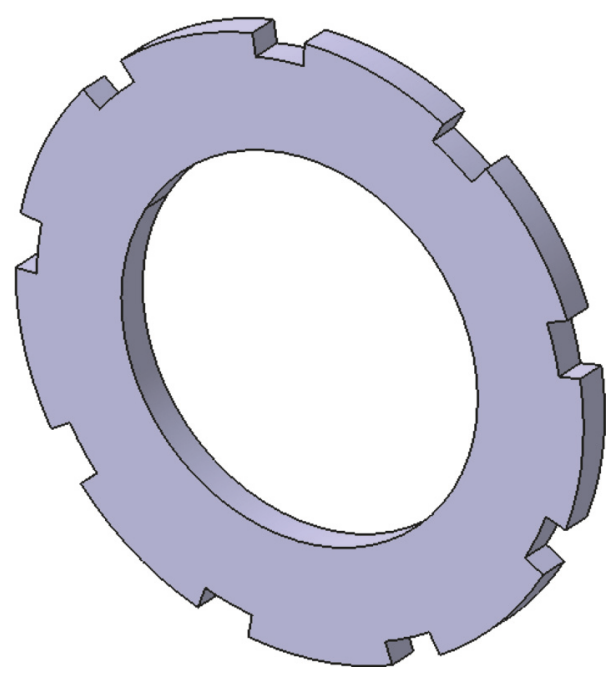

Figure 3: Simplified model of rotor.

the contact surface is 0.3 . Because straggling brake discs are the key objects in the analysis, several tiny holes, fillets, and chamfers are simplified or removed to ensure a more qualified mesh. Finished discs models are shown in Figures 2-4.

Import these simplified disc models into ABAQUS software and construct the assembly model according to their actual position. The completed assembly model is shown in Figure 5.

2.2. Improvements of the Model. During brake procedure, contact behavior exists in multiple positions. Simulating contact behavior both conveniently and accurately is the key to analyzing brake squeal problem. Construct surface-surface contact pair on every contact surface of the brake disc, so friction effect can be built in the software. Contact property includes penalty function, so coefficient of friction can be

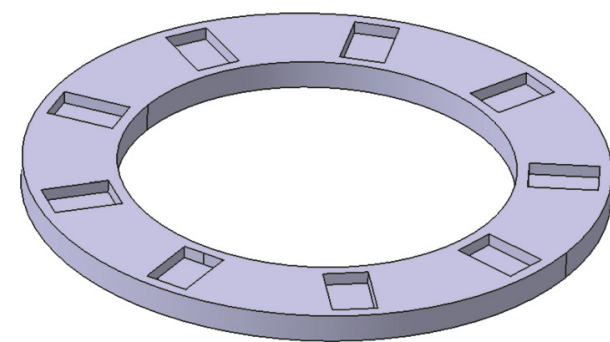

FIGURE 4: Simplified model of baseplate.

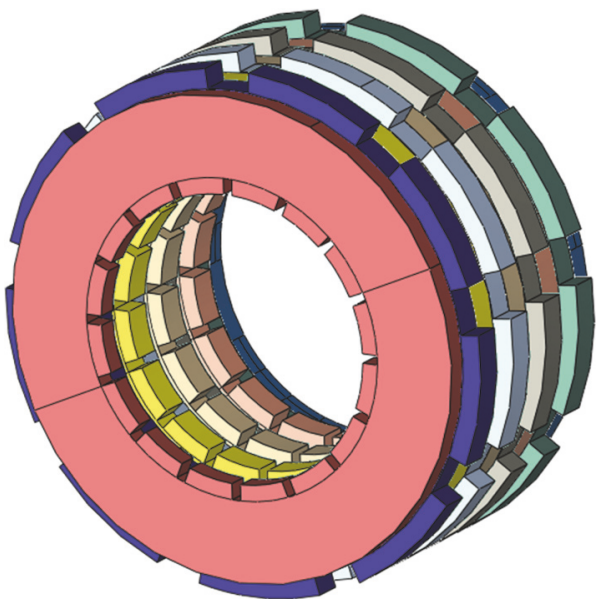

Figure 5: FEM model of assembly.

introduced. There are two kinds of contact algorithms in ABAQUS software, which are small sliding and finite sliding. Small sliding algorithm is applied, because subsequent analysis does not contain large scale of displacement compared with the size of element.

In order to satisfy accuracy and time consumption requirements of FEM simulation, partitions of the entire model are divided based on geometry appearance of the model. Afterwards, an exquisite structural mesh can be established. Comparison between directly generated mesh and partition based mesh is shown in Figure 6. It is obvious that, by setting up partitions, mesh becomes neater and more concise, which provides great accuracy and efficiency for further analysis. There are 11440 elements on rotor model, 11165 elements on stator model, and 8136 elements on baseplate model.

Finally, verify the entire mesh condition for excessive distortion for excessive errors. At last, FEM model of aircraft brake is finished.

\section{Complex Mode Analysis}

After finishing constructing of the FEM model of the brake system, subsequent squeal analysis can be performed. This chapter concentrates on complex mode theory, unstable vibration modes are extracted, and unstable vibration modes are analyzed. 

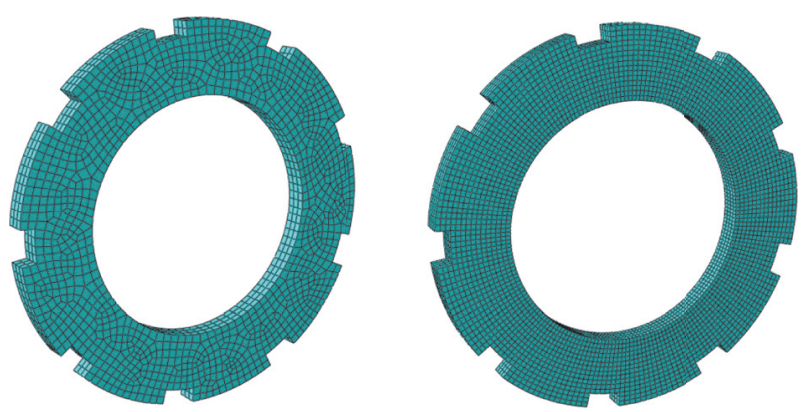

FiguRE 6: Comparison between different mesh strategies.

3.1. Theory of Complex Mode Analysis. In order to research brake squeal problem, modal analysis must be conducted to discover intrinsic vibration property of the entire model. Modal analysis means decoupling the differential equations which demonstrate vibration property of the system and transform physical coordinate into modal coordinate so relative modal parameters can be deduced.

As for ordinary real mode vibration analysis, mass matrix and stiffness matrix are both symmetric and positive definite. Damping matrix is a linear representation of mass and stiffness matrix, which can be decoupled. Moreover, natural vibration modes are real vectors which are orthogonal to mass, stiffness, and damping matrices. Therefore, modal coordinate is capable of simplifying the differential equations. However, in the aircraft disc brake model, damping matrix cannot be linearly represented by mass and stiffness matrices. As a result, modal parameters turn to be complex instead of real.

For an ordinary damped vibration system, damping force is in direct proportion to velocity. The vibration differential equation is

$$
M \ddot{X}+C \dot{X}+K X=F,
$$

where $M, C, K, X$, and $F$ are all matrices, representing mass, damping, stiffness, displacement, and force matrices. This equation can also be illustrated in matrix form.

$$
\left[\begin{array}{cc}
C & M \\
M & 0
\end{array}\right]\left\{\begin{array}{l}
\dot{X} \\
\ddot{X}
\end{array}\right\}+\left[\begin{array}{cc}
K & 0 \\
0 & -M
\end{array}\right]\left\{\begin{array}{l}
X \\
\dot{X}
\end{array}\right\}=\left\{\begin{array}{l}
F \\
0
\end{array}\right\} \text {. }
$$

Assume $2 N \times 1$ state vector $\bar{X}$ is

$$
\bar{X}=\left\{\begin{array}{c}
X \\
\dot{X}
\end{array}\right\} .
$$

So (2) can be simplified as

$$
\begin{aligned}
A \dot{\bar{X}}+B \bar{X} & =F, \\
A & =\left[\begin{array}{cc}
C & M \\
M & 0
\end{array}\right], \\
B & =\left[\begin{array}{cc}
K & 0 \\
0 & -M
\end{array}\right] .
\end{aligned}
$$

Equation (4) is the state equation of complex mode analysis. Matrix $A$ and matrix $B$ are both of $2 N \times 2 N$ size. For free vibration, $F$ becomes zero matrix. Assume root of the equation is

$$
\begin{aligned}
& X=\psi e^{\lambda t}, \\
& \dot{X}=\psi \lambda e^{\lambda t},
\end{aligned}
$$

where $\psi$ is

$$
\psi=\left[\phi_{1}, \ldots, \phi_{N}\right] .
$$

So (4) can be solved, and $2 \mathrm{~N}$ eigenvalues and eigenvectors can be acquired.

$$
\begin{gathered}
\lambda_{1}, \lambda_{1}^{*}, \lambda_{2}, \lambda_{2}^{*}, \ldots, \lambda_{N}, \lambda_{N}^{*}, \\
\left\{\begin{array}{c}
\psi_{1} \\
\psi_{1} \lambda_{1}
\end{array}\right\},\left\{\begin{array}{c}
\psi_{1}^{*} \\
\psi_{1}^{*} \lambda_{1}^{*}
\end{array}\right\}, \ldots,\left\{\begin{array}{c}
\psi_{N} \\
\psi_{N} \lambda_{N}
\end{array}\right\},\left\{\begin{array}{c}
\psi_{N}^{*} \\
\psi_{N}^{*} \lambda_{N}^{*}
\end{array}\right\} .
\end{gathered}
$$

Plug eigenvectors to original vibration equation (4),

$$
(A \lambda+B) \bar{\psi}=0, \quad \bar{\psi}=\left\{\begin{array}{c}
\psi \\
\psi \lambda
\end{array}\right\}
$$

for No. $r$ mode; it can be shown as

$$
\left(A \lambda_{r}+B\right) \bar{\psi}_{r}=0 .
$$

Combine with eigenvector of No. $s$ mode. It can be inferred that

$$
\begin{aligned}
& \bar{\psi}_{s}^{T}\left(A \lambda_{r}+B\right) \bar{\psi}_{r}=0, \\
& \bar{\psi}_{r}^{T}\left(A \lambda_{s}+B\right) \bar{\psi}_{s}=0 .
\end{aligned}
$$

Because $A$ and $B$ are both symmetric,

$$
\begin{aligned}
& \bar{\psi}_{r}^{T} A \bar{\psi}_{s}=\bar{\psi}_{s}^{T} A \bar{\psi}_{r}, \\
& \bar{\psi}_{r}^{T} B \bar{\psi}_{s}=\bar{\psi}_{s}^{T} B \bar{\psi}_{r} .
\end{aligned}
$$

So, it is evident that

$$
\bar{\psi}_{r}^{T} A \bar{\psi}_{s}\left(\lambda_{r}-\lambda_{s}\right)=0 .
$$

Therefore, orthogonal property of complex mode analysis is

$$
\begin{aligned}
& \bar{\psi}_{s}^{T} A \bar{\psi}_{r}=0, \\
& \bar{\psi}_{s}^{T} B \bar{\psi}_{r}=0 .
\end{aligned}
$$

$\lambda_{r}$ is the complex modal frequency of the vibration system. $\bar{\psi}_{r}$ is the complex modal eigenvector. These two parameters always come in conjugate pairs. According to the complex mode properties, a system with $N$ degrees of freedom has $2 N$ eigenvalues and eigenvectors. 
3.2. FEM Analysis with Complex Mode Method. It is vital to calculate undamped vibration frequency and vibration modes before any complex frequency analysis in ABAQUS software. Besides, static analysis step must be added to exert actual working conditions on the entire model, such as rotation speed and pressure, so accuracy can be ensured. Therefore, analysis steps of the entire simulation are listed below.

Step one is the static mechanic analysis. By exerting pressure on outward stator surface, brake force can be simulated, and contact interactions among parts are established. Moreover, stress status under actual working condition can be achieved. Stress may alter the stiffness matrix and influence subsequent frequency simulation.

Step two is generating rotating velocity. In ABAQUS static mechanic analysis, there are no rotating freedoms for nodes of $3 \mathrm{D}$ solid objects, so it is unable to exert rotation on rotors as boundary condition as usual. So, special keywords must be added to the model to solve this problem. Categorize every node of rotors under one set, named as "rotor." Then use keywords "* Motion, Rotation" to establish relevant rotation axis and velocity.

Step three is the extraction of natural frequency of undamped model. This step is the foundation of complex mode analysis.

Step four is the complex mode analysis. Complex frequency and complex eigenvalues are calculated and recorded.

Complex eigenvalues are all complex numbers. The imaginary part of complex eigenvalue indicates the complex vibration frequency, while real part of eigenvalue denotes stability of vibration. Complex modal damping equals to opposite number of real part divided by imaginary part. Therefore, a positive real part means a negative modal damping, which ultimately leads to unstable vibration.

There is another factor that needs to be taken into consideration. The existing material damping and structural damping are not included in the model, so total damping effect in the vibration system is smaller than it should be; this may cause the result to be more unstable. As a result, considering existing practical analysis experience, vibration modes with a modal damping less than -0.01 are determined to be unstable complex modes, which lead to brake squeal phenomenon.

In this analysis, eigenvalue data are extracted by history output and mapped in Figure 7.

The slope in the figure represents a -0.01 damping. Dots above the slope mean unstable vibration mode. Corresponding frequency can be seen in the figure as well. There are 36 unstable vibration modes in the analysis totally.

There has been no certain conclusion that the stronger squeal is, the more serious negative damping exists. And theoretical improvement of the relationship between squeal extent and negative damping value has not been conducted yet. However, based on past experiment experience and complex mode simulation results, the tendency of serious brake squeal phenomenon does relate to the real part value of corresponding eigenvalue. Thus it is practical to determine the brake squeal extent by analyzing real part of the eigenvalue.

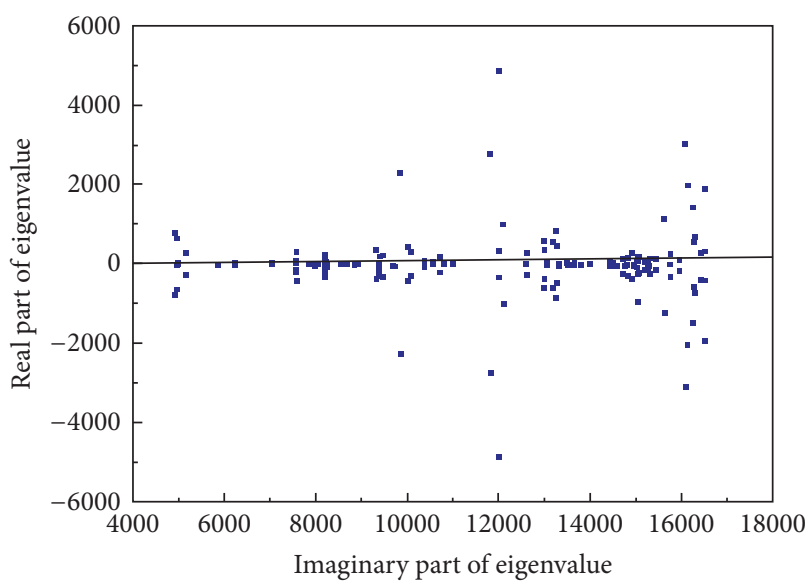

Figure 7: Complex eigenvalue scattergram.

In the analysis conducted in this chapter, unstable vibration mode firstly emerges at $4930 \mathrm{~Hz}$. It is clear that unstable modes do not distribute uniformly. In fact, they cluster around several certain values. $5 \mathrm{kHz}, 8 \mathrm{kHz}, 10 \mathrm{kHz}, 12 \mathrm{kHz}$, $13.5 \mathrm{kHz}$, and $16.2 \mathrm{kHz}$ are intervals where most unstable modes exist. As a result, the frequencies of brake squeal also exist in those intervals. So far, the frequency of brake squeal phenomenon has been calculated from complex mode analysis.

3.3. Properties of Unstable Vibration Modes. To take a deeper look at the unstable vibration property, it is vital to analyze specific orders of unstable vibration modes.

There are two major vibration mode categories, in-plane mode and out-of-plane mode [19]. According to past research experience, each mode is mainly dominated by only one category of vibration, while the other category has little effect.

Vibration modes dominated by out-of-plane motion possess obvious out-of-plane vibration tendency. This tendency can be described by static diameter and static circle. As for modes dominated by in-plane motion, circumferential direction and radial direction are analyzed. There will at least be one static diameter along circumferential direction motion, while there is no static diameter or static circle along radial direction. Some typical unstable vibration modes are shown in Figure 8. Contour shows the displacement along its major direction of every vibration mode. $F_{r}$ represents the real part of corresponding eigenvalue. It is noteworthy that the value of displacement has been normalized so it does not reflect any actual length.

Properties of unstable vibration modes can be concluded from the figures. Most of the unstable modes are dominated by out-of-plane vibration; only a few exceptions exist. Among these exceptions, radial direction vibration plays the main character. In terms of vibration modes whose frequency is under $10 \mathrm{kHz}$, brake discs have similar vibration tendency. On the other hand, if vibration frequency exceeds $10 \mathrm{kHz}$, different brake discs vibrate differently, with more static diameters. Moreover, brake discs near the baseplate vibrate fiercer under high frequency. 


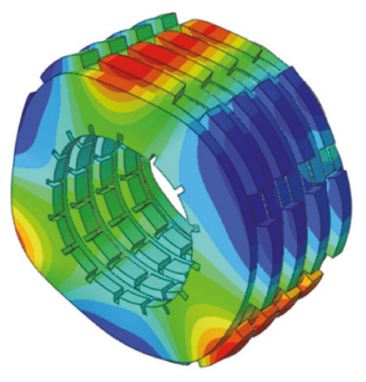

$$
f=4971 \mathrm{~Hz}, f_{r}=649
$$

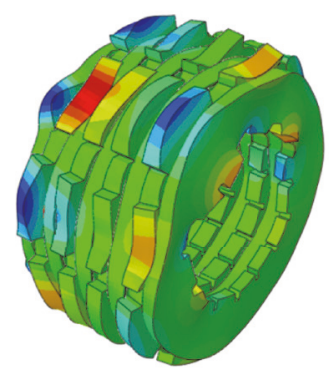

$f=12010 \mathrm{~Hz}, f_{r}=4727$
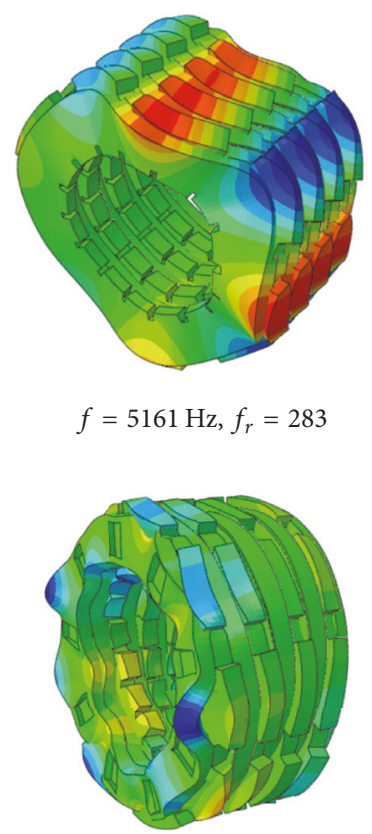

$f=13185 \mathrm{~Hz}, f_{r}=564$
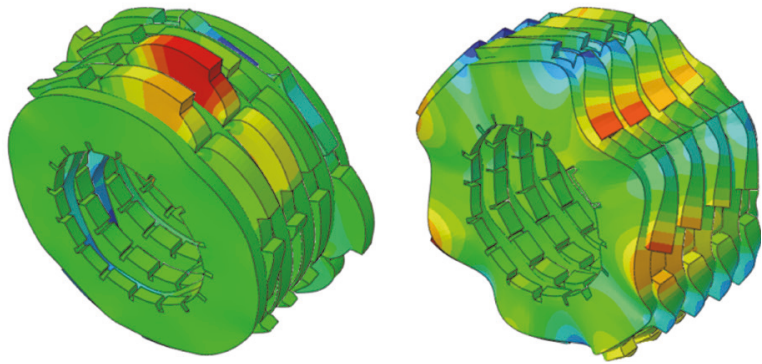

$$
f=7572 \mathrm{~Hz}, f_{r}=297
$$

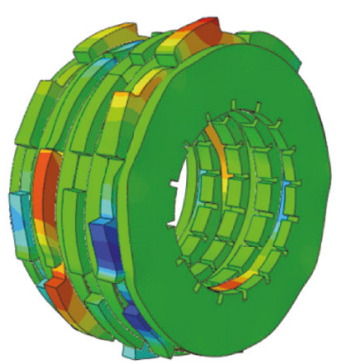

$f=13269 \mathrm{~Hz}, f_{r}=821$

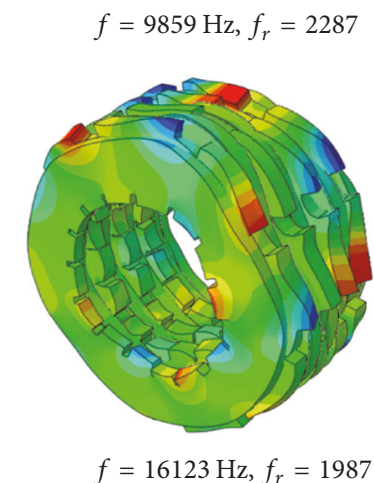

Figure 8: Typical unstable vibration modes.

\section{Transient Dynamic Analysis}

In order to study brake squeal problem more precisely, nonlinear properties must be taken into consideration. Explicit dynamic method is applied for transient dynamic analysis on brake squeal problem. Fourier transformation is also applied to analyze vibration property in the frequency domain.

4.1. Theory of Explicit Dynamic. Explicit dynamic analysis focuses on differences about time. No simultaneous equations need to be constructed or solved. Convergence is not a problem either. Dynamic analysis always offers a result, no matter how rough the model is. However, the increment step length is heavily influenced by the FEM element quality. The number of increment steps is also very tremendous, so it takes a lot of time and resource to conduct the analysis.

ABAQUS applies central difference method for solving transient dynamic problems. The FEM process can be shown as follows:

$$
M \ddot{x}^{(t)}=f_{\text {ex }}^{(t)}-f_{\text {in }}^{(t)} .
$$

In the equation, $\ddot{x}$ stands for acceleration vector and $M$ is the mass matrix. $f_{\text {ex }}$ represents external load, and $f_{\text {in }}$ represents internal load. During explicit simulations, there are certain relations between velocity and displacement:

$$
\begin{aligned}
\dot{x}^{(t+0.5 \Delta t)} & =\dot{x}^{(t-0.5 \Delta t)}+\frac{\Delta t^{(t+\Delta t)}+\Delta t^{(t)}}{2} \ddot{x}^{(t)}, \\
x^{(t+\Delta t)} & =x^{(t)}+\Delta t^{(t+\Delta t)} \dot{x}^{(t+0.5 \Delta t)} .
\end{aligned}
$$

In the equation, $t \pm 0.5 \Delta t$ stands for the middle increment step during calculations and $\Delta t$ is the step length. Difference, instead of differential, is applied in explicit dynamic analysis, so step length must be short enough to ensure sufficient accuracy.

4.2. Explicit Dynamic Analysis. Explicit analysis step is unable to coexist with previous static analysis steps, so a whole new set of analysis steps are needed. During the linear perturbation steps in the last chapter, the entire system is stable in every increment step, and time does not participate in the actual simulation, while, in dynamic analysis, time is an important parameter which heavily influences the analysis procedure.

Therefore, the new analysis steps need further modification. Keywords are not able to exert rotation to the rotors, so a reference point is established at the axis of rotation. Then couple every node of rotor model with the reference point, so that boundary conditions can be exerted at the reference point as well as the rotors. Rotation and brake force are both gradually applied according to time, as shown in Figure 9.

After finishing applying rotation speed, simulation continues for 0.01 seconds to observe and analyze the vibration, which grants a $100 \mathrm{~Hz}$ lower limit for frequency domain analysis.

Change contact algorithm into finite sliding method, because the relative sliding is large compared to the size of single element. According to the purpose of the transient dynamic analysis, extra history output must be added. Pick a certain observation point on the rotor and record the displacement data every $5 \mathrm{~ms}$, which ensures a high sampling density. After completing the simulation, map the curves of displacements along three directions, as shown in Figures 10 and 11. In this analysis, $X$ is the axial direction and $Y$ and $Z$ are 


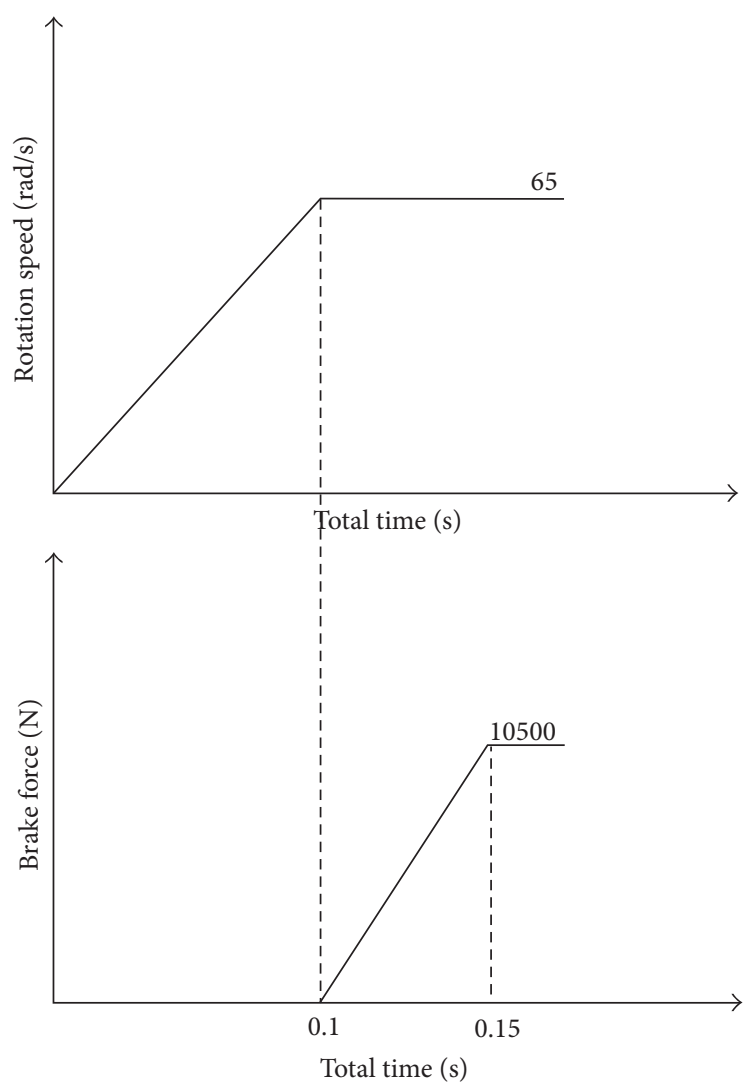

FIGURE 9: Applying process of rotation speed and brake force.

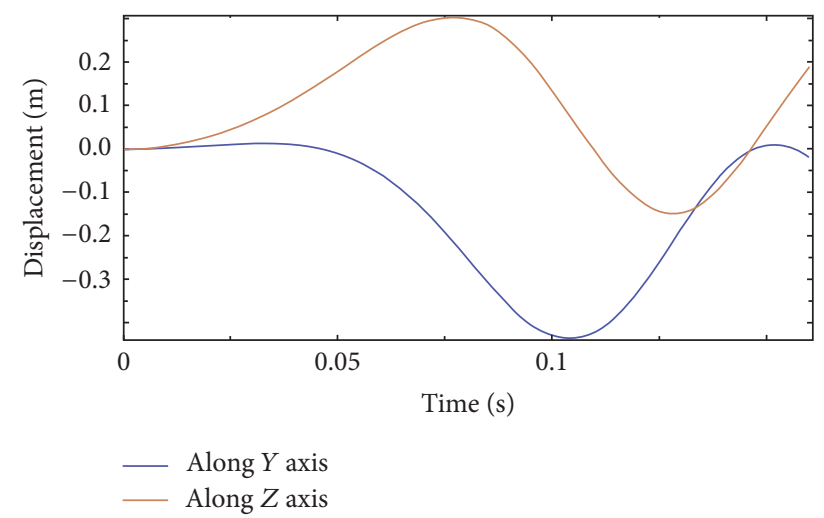

Figure 10: Displacement curves of $Y$ and $Z$ direction.

the directions in the disc plane. In Figure 10, the blue curve indicates displacement along $Y$ direction and brown curve reflects $Z$ direction.

From these two figures, it can be concluded that rotation dominates the motion along $Y$ and $Z$ axis; no obvious high frequency vibration takes place. This matches the result from complex mode analysis, where in-plane unstable vibration modes are minority. In terms of $X$ axis direction, the amplitude of the displacement is merely among millimeters, which is very tiny compared with the size of brake disc. At 0.01 seconds when brake force is applied, high frequency vibration takes place. As brake force gradually increases, the vibration

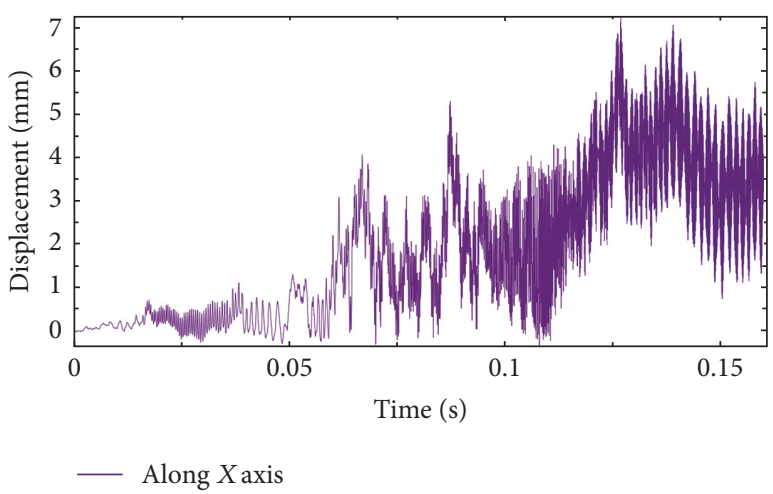

Figure 11: Displacement curve of $X$ direction.
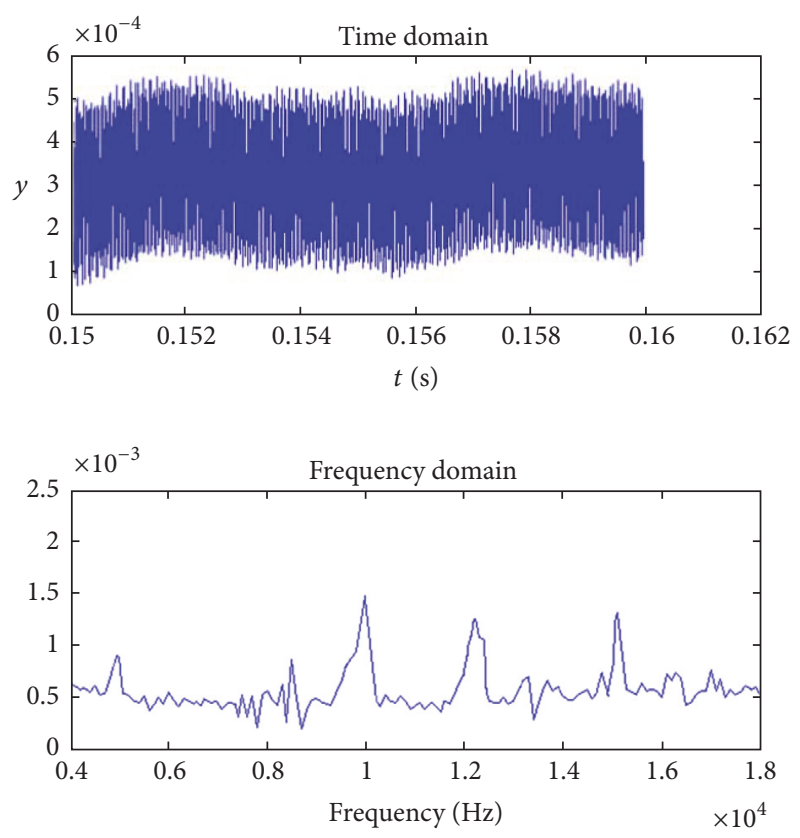

FIGURE 12: Displacement curves in time and frequency domain.

becomes more acute. To study the vibration property in frequency domain, extract the last 0.01 -second curve and then use MATLAB to perform Fourier transformation; then Figure 12 can be achieved.

From Figure 12 it is evident that acute vibration takes place around $5 \mathrm{kHz}, 8 \mathrm{kHz}, 10 \mathrm{kHz}, 12 \mathrm{kHz}$, and $15 \mathrm{kHz}$ intervals. By comparing this result with result from last chapter, it can be concluded that squeal phenomenon around $5 \mathrm{kHz}$, $10 \mathrm{kHz}$, and $12 \mathrm{kHz}$ has been accurately predicted by complex mode analysis, while $8 \mathrm{kHz}, 13.5 \mathrm{kHz}$, and $15 \mathrm{kHz}$ vibration frequency do not match the result from complex mode analysis. These phenomena are called "overprediction" and "missing order" by other researchers. The reason for such phenomena lies in the linear method used by complex mode analysis. As a linear perturbation analysis step, complex mode analysis uses linear algorithm to approach nonlinear problem in its essence. Although it saves a lot of time and resource, inaccuracy is inevitable. 


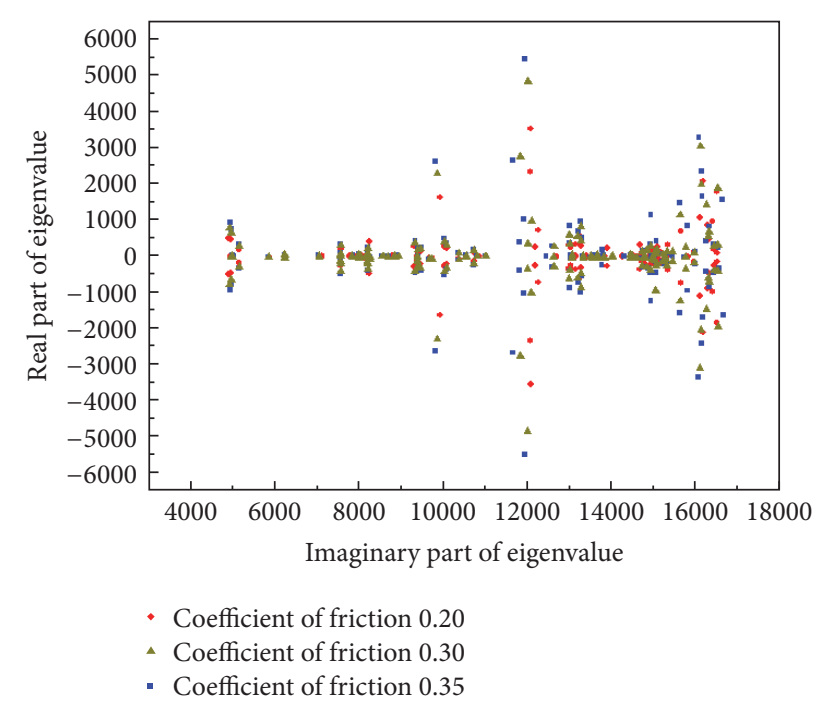

FIgURE 13: Complex eigenvalue scattergram of different coefficient of friction.

On the other hand, explicit dynamic analysis takes nonlinearity into consideration, so accuracy is guaranteed. Nevertheless, explicit dynamic analysis requires a considerable amount of time and resource. For instance, the simulation in this chapter is conducted using 16 cores of high-performance $\mathrm{CPU}$ and takes more than two hours to finish. Complex mode analysis merely takes less than 20 minutes to complete. As a result, explicit dynamic method is not practical to conduct squeal parameter study.

As a result, though complex mode analysis is less accurate than explicit dynamic method, it is still a handy tool to analyze brake squeal problem. Complex mode analysis consumes moderate resource and time and provides satisfactory accuracy, which is enough for subsequent simulations.

\section{Parameter Study of Brake Squeal}

As is analyzed in previous chapters, brake squeal is caused by friction-induced unstable modal coupling. Thus changing physical properties of the brake discs may be an effective way to ease the squeal phenomenon. In this chapter, three parameters are analyzed using complex mode analysis method.

5.1. Coefficient of Friction. After modifying the penalty function of the contact property, the effect of different coefficients of friction can be analyzed.

In Figure 13 it can be discovered that, as the coefficient of friction increases, the real part of complex eigenvalue rises as well, which means vibration becomes more unstable. Moreover, the increase of coefficient of friction also slightly alters the distribution of vibration frequency.

So, according to the analysis result, to ease the brake squeal phenomenon, coefficient of friction has to be as low as possible. However, a low coefficient of friction weakens the brake performance, which makes it difficult to stop the aircraft, because the brake time and brake length cannot be

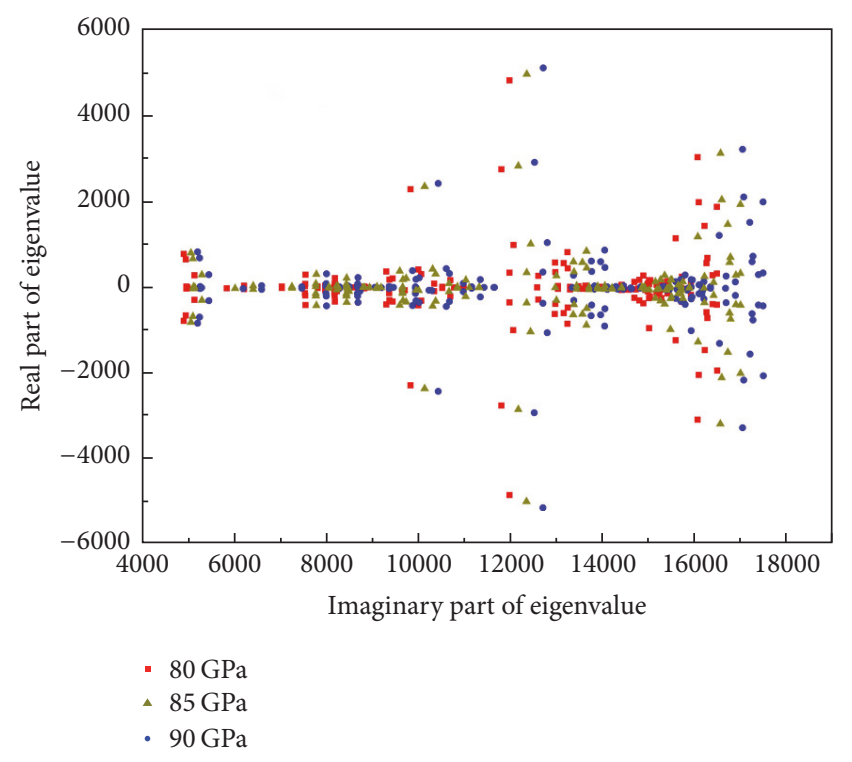

FIGURE 14: Complex eigenvalue scattergram of different stiffness.

extended at will. As a result, altering coefficient of friction is not a suitable option to solve the brake squeal problem.

5.2. Stiffness of Brake Disc. Stiffness matrix of the vibration equation gets changed while stiffness of brake disc changes. Let Young's modulus be $80 \mathrm{GPa}, 85 \mathrm{GPa}$, and $90 \mathrm{GPa}$, respectively, map the complex eigenvalue scattergram in Figure 14.

It can be concluded that vibration frequency rises as the stiffness increases, which matches the common sense. The real part values have a positive correlation with stiffness, which means the harder the brake discs become, the more easily they squeal. So changing the stiffness of the brake disc for the lower is an auxiliary option for solving brake squeal problem and lowering the squeal frequency.

5.3. Brake Force Fluctuation. During taxiing procedure, the carbon brake discs have a complicated effect on the entire brake system [20-22]. For example, assembling clearance [23], eccentric wear, and wear particles may cause considerable brake force fluctuation. The fluctuation brings extra excitement for the vibration system, which heavily influences brake system performance. Steady-state dynamic analysis has been performed to study the effect of brake force fluctuation.

Steady-state dynamic is an important category of dynamic analysis. It focuses on researching dynamic response under steady simple harmonic load. By performing steady-state dynamic analysis, it is able to verify the system's ability to tolerate resonance and fatigue. Steady-state dynamic aims at acquiring the relationship between displacement and the frequency of external excitement, so subsequent structural optimization and modification can be carried out. Only steady-state response will be recorded and analyzed in steady-state analysis.

Delete original natural frequency and complex frequency analysis steps and set up a direct steady-state analysis step. Establish a load whose amplitude is $2 \mathrm{kN}$ and use default 


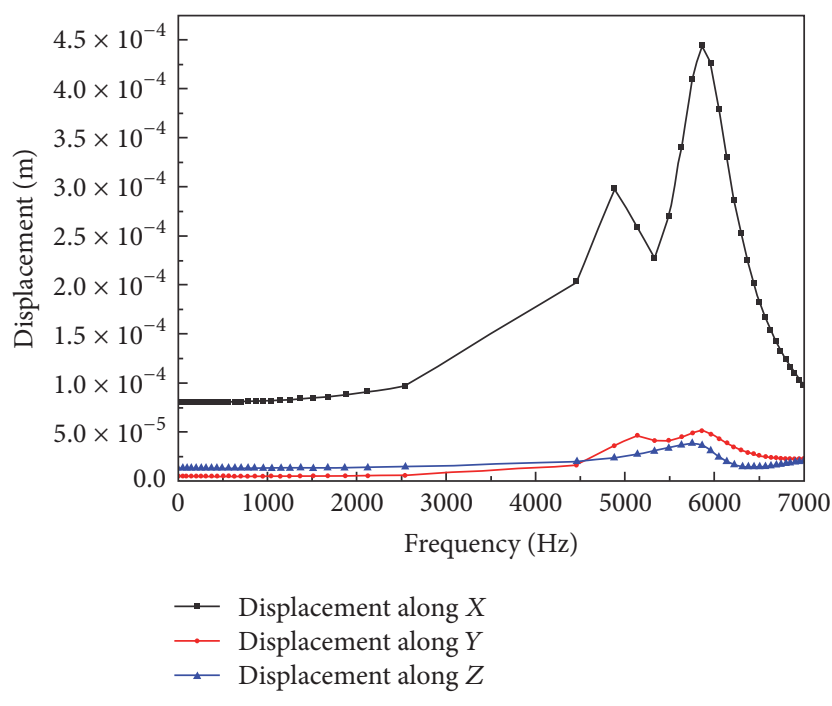

FIGURE 15: Steady-state displacement response and excitement frequency.

simple harmonic function to exert this load for steady-state analysis. The frequency range of the load is under $7 \mathrm{kHz}$.

After completing the simulation, extract displacement curves about frequency on the observation point, as shown in Figure 15.

It is obvious that displacement along $X$ direction plays a main character compared to other directions. While the frequency of brake force excitement is under $2500 \mathrm{~Hz}$, the steady-state displacement response is stable. At $4880 \mathrm{~Hz}$, first displacement peak takes place, which is also the first unstable mode according to complex mode analysis. Between $4880 \mathrm{~Hz}$ and $5859 \mathrm{~Hz}$, steady-state displacement response along $Y$ and $Z$ direction rises. As a result, external excitement fluctuation must be limited under $4880 \mathrm{~Hz}$ to prevent from acute vibration, which leads to severe squeal phenomenon.

\section{Vibration Reduction Using Viscoelastic Damping Material}

6.1. Vibration Reduction Principle Based on Viscoelastic Damping. According to the results above, modifying coefficient of friction, stiffness of brake disc, and brake force fluctuation frequency can improve brake squeal problem. But modifying coefficient of friction, stiffness is enslaved to the internal characteristics of carbon disc and the operability is limited. Brake force fluctuation frequency is related to many coefficients, such as manufacturing and installation error, using clearance, microscopic characteristics of disc surface, and so on. It is difficult to control the brake force fluctuation frequency. The viscoelastic damping material was introduced to the brake disc manufacturing and installation in the automotive industry, which has a good effect on the brake squeal problem. So the vibration reduction effect will be studied by using the viscoelastic damping material to the aircraft brake disc.
Dissipation factor is used to describe the damping. As the shearing deformation expends more energy than tensile deformation, the dissipation factor produced by complex shear modulus $G$ is studied here.

$$
\begin{aligned}
G & =G^{\prime}+j G^{\prime \prime}=G^{\prime}(1+j \tan \alpha), \\
\tau & =\left(G^{\prime}+j G^{\prime \prime}\right) \gamma,
\end{aligned}
$$

where $G^{\prime}$ is the real part of the complex shear modulus and $G^{\prime \prime}$ is the imaginary part of the complex shear modulus. $\alpha$ is the phase difference of the strain and stress after excitation. $\tau$, $\gamma$ are the shear stress and shear strain. Shear dissipation factor $\beta$ can be described by

$$
\beta=\frac{G^{\prime \prime}}{G^{\prime}}=\tan \alpha .
$$

The physical meaning of $\beta$ is the ratio of the dissipation energy $\Delta W$ and the stored energy $W$ by elastic deformation. Suppose the material excited by sine force. Because of hysteretic phenomena, $\Delta W$ can be derived:

$$
\Delta W=\int_{0}^{2 \pi} \tau d \gamma=\tau_{0} \gamma_{0} \pi \sin \alpha,
$$

where $\tau_{0}, \gamma_{0}$ are amplitude of shear stress and shear strain, respectively. Because

$$
\begin{aligned}
G^{\prime} & =\frac{\tau_{0}}{\gamma_{0}} \cos \alpha, \\
G^{\prime \prime} & =\frac{\tau_{0}}{\gamma_{0}} \sin \alpha,
\end{aligned}
$$

the dissipation energy $\Delta W$ is

$$
\Delta W=\pi G^{\prime} \beta \gamma_{0}^{2} .
$$

From formula (20) the key point to reduce vibration using the viscoelastic damping material is to maximize the product of the real part of the complex shear modulus $G^{\prime}$ and shear dissipation factor $\beta$. The two coefficients are affected by temperature and vibration frequency, so the ability of vibration reduction fluctuates under different work conditions and vibration frequency. The real part of the complex shear modulus $G^{\prime}$ and shear dissipation factor $\beta$ can be determined by test $[24,25]$.

Considering the structure of the aircraft brake disc and work conditions, a ring-shaped layer of viscoelastic damping material is constrained to outer margin of the stator. Shear strain of the ring-shaped damping layer can absorb and dissipate the energy of the out-of-plane vibration to improve the brake squeal problem. The multiphase iron carbon alloy is used as the viscoelastic damping material in this paper. Because the dissipation factor $\beta$ of the material cannot be directly defined in the software ABAQUS, the Rayleigh damping is used to define the damping. Under the circumstance of the material used in this paper, the dissipation factor satisfies

$$
\begin{aligned}
\eta & =2 \zeta=\frac{\Delta}{\pi}=Q^{-1}=\frac{\mathrm{SDC}}{200 \pi}, \\
\frac{a}{2 \omega}+\frac{b \omega}{2} & =\xi,
\end{aligned}
$$




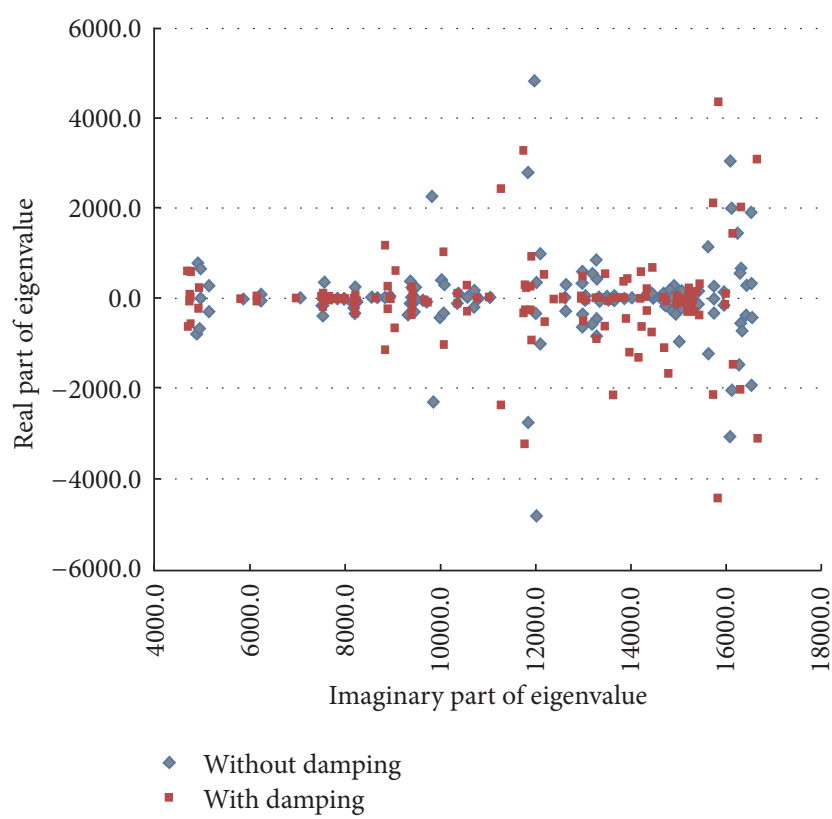

FIGURE 16: Complex eigenvalue scattergram comparison with or without damping.

where $\zeta$ is the damping ratio, $\Delta$ is the logarithm deletion rate, $Q$ is quality factor, and SDC is the specific damping capacity. SDC of the multiphase iron carbon alloy is about $20 \%$, so the damping ratio $\zeta$ can be derived by formula (21): 0.000159. $a$ and $b$ are the mass matrix coefficient and stiffness matrix coefficient, respectively. The CAE model of the ring-shaped damping layer is established separately and the normal frequency is computed. The first two frequencies are extracted, using formula (22); the Rayleigh damping factor can be obtained approximately. Then this factor is used to the complex eigenvalue analyzing.

6.2. Results of Vibration Reduction. After the ring-shaped damping layer constrained to outer margin of the stator and the damping factor set-up, the disc complex modal is computed and the complex eigenvalues distribution is obtained in Figure 16.

Under the effect of damping, the imaginary part of eigenvalue of every rank changes remarkably, which indicates the vibration frequency of the damping system changes. Meanwhile, the real part of eigenvalue decreases mostly. But in a few ranks, new unstable modal is produced by the damping material, so the damping method needs to be optimized further. In general, damping material changes the vibration nature and can restrain the unstable modal evidently. It is also an operable and economy method to improve the brake squeal problem.

\section{Conclusions}

(1) Aircraft brake noise problem contains a lot of nonlinear properties, which makes it a sophisticated engineering issue. A disc brake FEM model based on actual aircraft brake has been established. By applying FEM analysis method, complex mode analysis has been performed on the brake model. The possibility of squeal has been analyzed according to the eigenvalues results, and vibration properties of typical unstable vibration modes which are highly likely to cause squeal have been studied thoroughly. Explicit dynamic method has been taken to conduct transient dynamic analysis. Brake disc's vibration properties have been researched in both time and frequency domain. Comparisons between these two methods have been performed. Complex mode method has been adopted for further parameter analysis.

(2) Parameter analysis has been conducted to study the influence of coefficient of friction, stiffness, and brake force fluctuation frequency on the brake squeal phenomenon. The results show that a small coefficient of friction, a low brake disc stiffness, or relatively low brake force fluctuation frequency all help improve the brake squeal problem.

(3) A ring-shaped layer of viscoelastic damping material is constrained to outer margin of the stator to restrain the unstable modal. The simulation results indicate that this method can change the vibration nature and improve the brake squeal problem.

\section{Conflicts of Interest}

The authors declare that there are no conflicts of interest regarding the publication of this paper.

\section{Acknowledgments}

This study was supported by the Fundamental Research Funds for the Central Universities (no. NS2016001), the National Natural Science Foundation of China (no. 51305198), and the Aero-Science Fund of China (no. 20142852025).

\section{References}

[1] J. J. Enright, "Reducing aircraft brake squeal with a damped brake-rod," in Proceedings of the World Aviation Conference, p. 5599, San Diego, Calif, USA, October 2000.

[2] M. Neubauer and R. Oleskiewicz, "Brake squeal control with shunted piezoceramics-efficient modelling and experiments," Proceedings of the Institution of Mechanical Engineers, Part D: Journal of Automobile Engineering, vol. 222, no. 7, pp. 1141-1151, 2008.

[3] D. Hochlenert, G. Spelsberg-Korspeter, and P. Hagedorn, "Friction induced vibrations in moving continua and their application to brake squeal," Journal of Applied Mechanics, vol. 74, no. 3, pp. 542-549, 2007.

[4] S. Y. Liu, J. T. Gordon, and M. A. Özbek, "Nonlinear model for aircraft brake squeal analysis: model description and solution methodology," Journal of Aircraft, vol. 35, no. 4, pp. 623-630, 1998.

[5] H. Hetzler, "Bifurcation analysis for brake squeal," in Proceedings of ASME 10th Biennial Conference on Engineering Systems Design and Analysis, ESDA '10, pp. 253-262, American Society of Mechanical Engineers, July 2010.

[6] H. D. Guan and X. D. Su, "An overview on brake vibration and noise," Engineering Mechanics, vol. 21, no. 4, pp. 150-155, 2004. 
[7] P. Z. Yu, X. D. Yin, and J. L. Zhang, "A review on brake judder," Automotive Engineering, vol. 27, no. 3, pp. 372-376, 2005.

[8] F. Bergman, M. Eriksson, and S. Jacobson, "The effect of reduced contact area on the occurrence of disc brake squeals for an automotive brake pad," Proceedings of the Institution of Mechanical Engineers, Part D: Journal of Automobile Engineering, vol. 214, no. 5, pp. 561-568, 2000.

[9] H. M. Lu, L. J. Zhang, and Z. P. Yu, "A review of automotive disc brake squeal," Journal of Vibration and Shock, vol. 40, no. 4, pp. $1-7,2011$.

[10] F. Chen, "Disc brake squeal: an overview," SAE Technical Papers 2007-01-0587, 2007.

[11] G. Spelsberg-Korspeter, "Eigenvalue optimization against brake squeal: symmetry, mathematical background and experiments," Journal of Sound and Vibration, vol. 331, no. 19, pp. 4259-4268, 2012.

[12] J. Z. Zhuo, W. D. Xie, and B. X. Ning, "An overview on disc brake vibrations and noise," Machinery Design \& Manufacture, vol. 11, pp. 215-217, 2007.

[13] O. Dessouki, G. Drake, B. Lowe, and W. K. Chang, "Disc brake squeal: diagnosis and prevention," SAE Technical Papers 200301-1618, 2003.

[14] F. Renaud, G. Chevallier, J.-L. Dion, and G. Taudire, "Motion capture of a pad measured with accelerometers during squeal noise in a real brake system," Mechanical Systems and Signal Processing, vol. 33, no. 2, pp. 155-166, 2012.

[15] S. Oberst and J. C. S. Lai, "Statistical analysis of brake squeal noise," Journal of Sound and Vibration, vol. 330, no. 12, pp. 29782994, 2011.

[16] D. M. Beloiu and R. A. Ibrahim, "Analytical and experimental investigations of disc brake noise using the frequency-time domain," Structural Control and Health Monitoring, vol. 13, no. 1, pp. 277-300, 2006.

[17] W. A. Wen, Complex eigenvalue analysis of the squeal propensity of a railway vehicle disc brake system using the finite element method [Ph.D. Thesis], Southwest Jiaotong University, Chengdu, China, 2007.

[18] H. Chen and L. Y. Chen, "Study on automobile disc brake brakes shaking problem," Internal Combustion Engine and Parts, no. 2, pp. 11-13, 2013.

[19] K. Chen, Experimental and computational research on disc brake squeal [Ph.D. Thesis], Harbin Institute of Technology, Harbin, China, 2014.

[20] T. J. Hutton, B. McEnaney, and J. C. Crelling, "Structural studies of wear debris from carbon-carbon composite aircraft brakes," Carbon, vol. 37, no. 6, pp. 907-916, 1999.

[21] L. Dagli and Y. Remond, "Identification of the non-linear behaviour a $4 \mathrm{D}$ carbon-carbon material designed for aeronautic application," Applied Composite Materials, vol. 9, no. 1, pp. 1-15, 2002.

[22] X. Xiong, B.-Y. Huang, J.-H. Li, and H.-J. Xu, "Friction behaviors of carbon/carbon composites with different pyrolytic carbon textures," Carbon, vol. 44, no. 3, pp. 463-467, 2006.

[23] G. Zhang, M. Xie, J. Li, G. Qi, and X. Pu, "Vehicle brake moan noise induced by brake pad taper wear," Journal of Mechanical Engineering, vol. 49, no. 9, pp. 81-86, 2013.

[24] X. T. Liu, M. Y. Shi, and X. H. Hua, "Current situation and prospect of active constrained layer damping vibration control technology," Journal of Vibration and Shock, vol. 20, no. 2, pp. $1-6,2001$.
[25] Q. Yang, X. Wang, W. W. Zhang et al., "Study on Noise Suppressing Performance of Constrained Layer Damping Structure," Journal of Noise and Vibration Control, no. 4, pp. 150-157, 2010. 


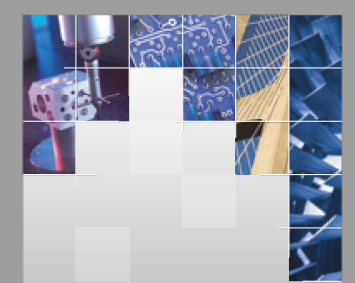

\section{Enfincering}
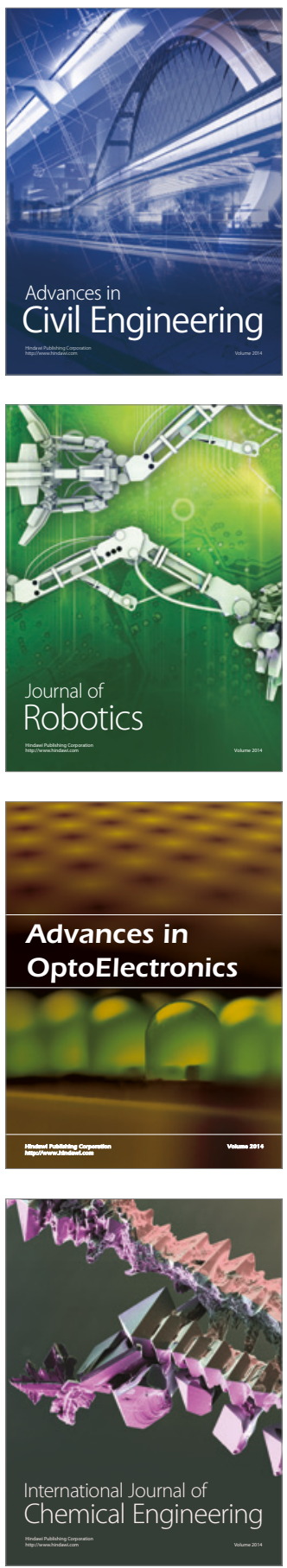

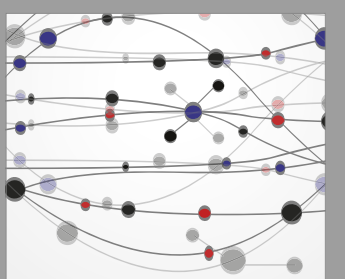

The Scientific World Journal

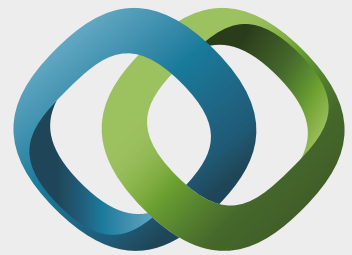

\section{Hindawi}

Submit your manuscripts at

https://www.hindawi.com
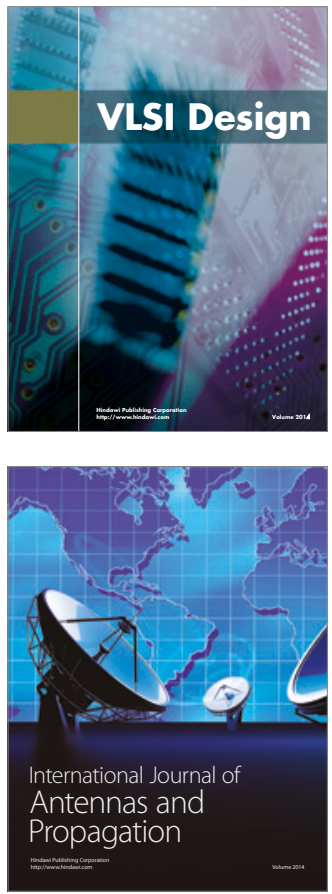

\section{Rotating}

Machinery
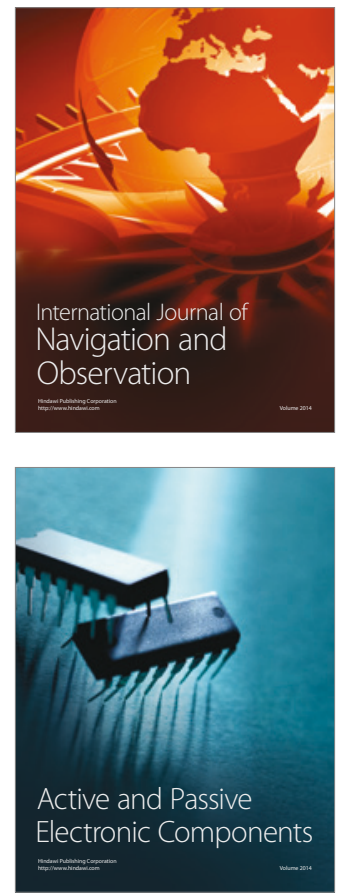
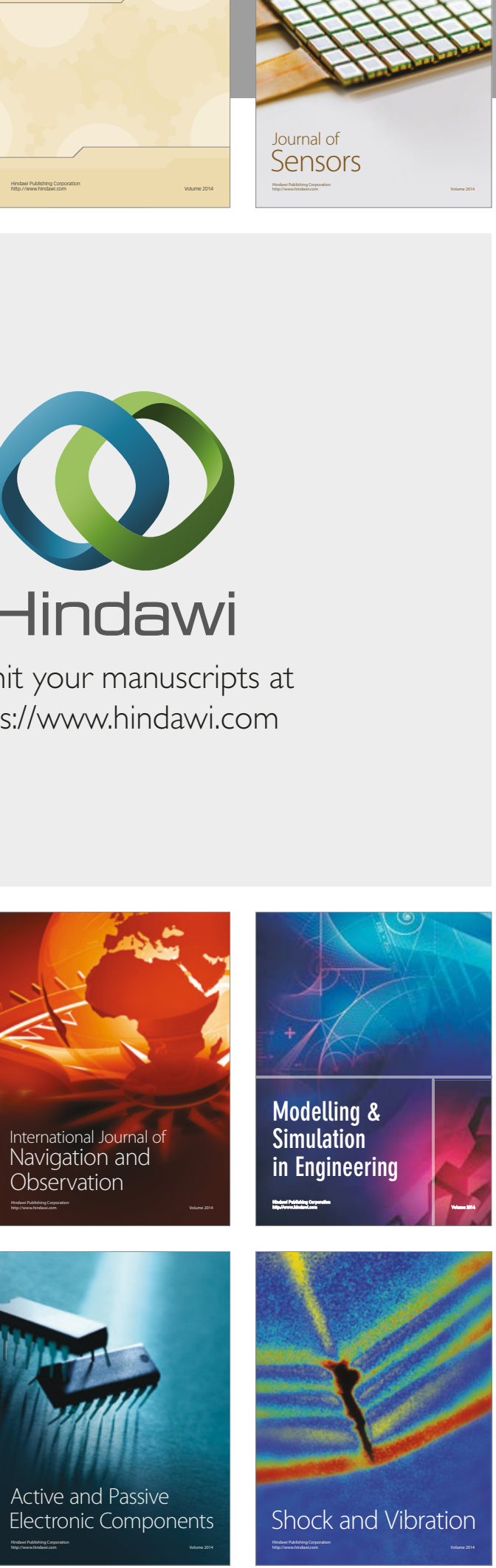
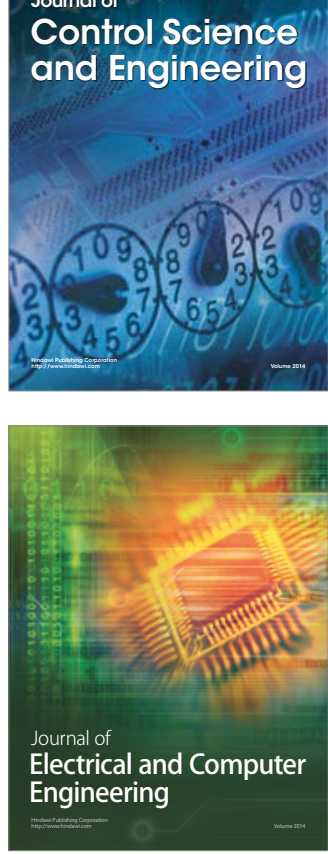

Distributed

Journal of

Control Science

and Engineering
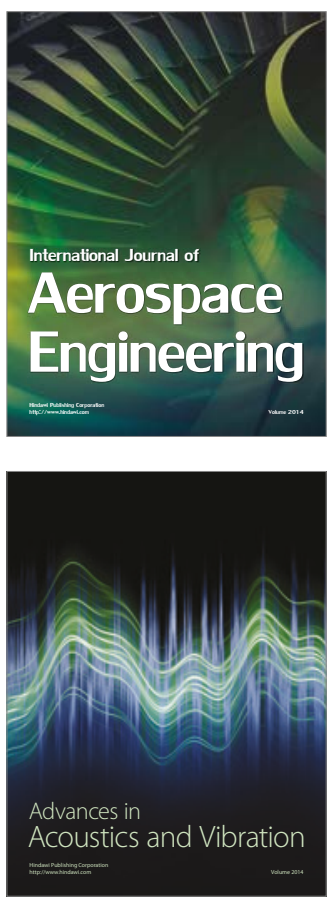

Sensor Networks 